International Journal of Geometric Methods in Modern Physics Vol. 16, No. 4 (2019) 1992002 (4pages)

(C) World Scientific Publishing Company

DOI: $10.1142 / \mathrm{S} 0219887819920026$

\title{
Erratum \\ Curvature properties of Gödel metric
}

[Int. J. Geom. Methods Mod. Phys. 11(3) (2014) 1450025]

\author{
Ryszard Deszcz \\ Department of Mathematics \\ Wroctaw University of Environmental and Life Sciences \\ Grunwaldzka 53, 50-357 Wroctaw, Poland \\ ryszard.deszcz@upwr.edu.pl \\ Marian Hotloś \\ Department of Applied Mathematics \\ Wroctaw University of Science and Technology \\ Wybrzeże Wyspiańskiego 27, 50-370 Wroctaw, Poland \\ marian.hotlos@pwr.edu.pl \\ Jan Jełowicki \\ Department of Mathematics \\ Wroctaw University of Environmental and Life Sciences \\ Grunwaldzka 53, 50-357 Wroctaw, Poland \\ jan.jelowicki@upwr.edu.pl \\ Haradhan Kundu* and Absos Ali Shaikh ${ }^{\dagger}$ \\ Department of Mathematics \\ University of Burdwan \\ Golapbag, Burdwan-713104, West Bengal, India \\ *kundu.haradhan@gmail.com \\ †aask2003@yahoo.co.in \\ Published 13 March 2019
}

We present some corrections of a part of the original paper [5. Sec. 4, lines $14^{9}-15^{9}$ ]. In addition, we give examples of manifolds related to the presented corrections.

Further, we denote by $S$ and $\kappa$ the Ricci tensor and the scalar curvature of the product manifold $(\bar{M} \times \widetilde{N}, g=\bar{g} \times \widetilde{g})$, respectively. It is obvious that $\operatorname{rank} S=$ $\operatorname{rank} \bar{S}$ and $\kappa=\bar{\kappa}$ on $U_{C} \subset \bar{M} \times \widetilde{N}$. Now [5 Eq. (24)] yields on this set

$$
\operatorname{rank}\left(S-\left(\frac{\kappa}{n-1}-(n-2) L_{C}\right) g\right) \leq 2 .
$$


We note that $\operatorname{rank}\left(S-\left(\frac{\kappa}{n-1}-(n-2) L_{C}\right) g\right)=1$ if and only if $\operatorname{rank} \bar{S}=1$ and $L_{C}=\frac{\kappa}{(n-2)(n-1)}$. Therefore the modified version of [5] Theorem 3] is the following theorem.

Theorem 0.1. Let $(\bar{M} \times \tilde{N}, g=\bar{g} \times \widetilde{g})$ be the product manifold of an $(n-1)$ dimensional semi-Riemannian manifold $(\bar{M}, \bar{g}), n \geq 4$, and a one-dimensional manifold $(\tilde{N}, \widetilde{g})$. Moreover, let $(\bar{M}, \bar{g})$ be a conformally flat manifold, provided that $n \geq 5$. If on $\bar{M}$ we have $\operatorname{rank}(\bar{S}-\rho \bar{g})=1$, for some function $\rho$, then $\operatorname{rank}(S-\rho g) \leq 2$ and [5, Eq. (12)], i.e. $C \cdot C=L_{C} Q(g, C)$, with $L_{C}=\frac{1}{n-2}\left(\frac{\kappa}{n-1}-\rho\right)$, hold on $\bar{M} \times \tilde{N}$. In particular, if the rank of the Ricci tensor $\bar{S}$ of $(\bar{M}, \bar{g})$ is one, then the rank of the Ricci tensor $S$ of $\bar{M} \times \widetilde{N}$ is also one and [5, Eq. (12)], with $L_{C}=\frac{\kappa}{(n-2)(n-1)}$, or equivalently, con $h(R) \cdot \operatorname{con} h(R)=0$ holds on $\bar{M} \times \widetilde{N}$.

We recall that semi-Riemannian manifolds satisfying the condition $\operatorname{rank}(S-$ $\rho g)=2$ are called 2-quasi-Einstein manifolds (see, e.g. [2 4]). We present now an application of the last theorem. From Theorem 4.1 of the paper [67] (cited in [5]), it follows that a hypersurface $M$ immersed isometrically in a semi-Riemannian space of constant curvature $N, \operatorname{dim} N \geq 5$, is a quasi-umbilical hypersurface if and only if it is a conformally flat manifold. Furthermore, using the Gauss equation of $M$ in $N$, we can easily prove that if $M$ is quasi-umbilical hypersurface then it is also a quasi-Einstein manifold. These facts, together with the last theorem, lead to the following theorem.

Theorem 0.2. Let $(\bar{M}, \bar{g})$ be a manifold which is isometric with a quasi-umbilical hypersurface immersed isometrically in a semi-Riemannian space of constant curvature $N, \operatorname{dim} N \geq 5$. Let $(\tilde{N}, \widetilde{g})$ be a one-dimensional manifold. Then the product manifold $(\bar{M} \times \tilde{\widetilde{N}}, g=\bar{g} \times \widetilde{g})$ is a manifold with pseudosymmetric Weyl conformal curvature tensor. Moreover, on the subset $U_{C}$ of this manifold we have $\operatorname{rank}(S-\rho g) \leq 2$, where $\rho$ is some function on $U_{C}$.

In this way, we obtain examples of quasi-Einstein and 2-quasi-Einstein manifolds [9, 10] with pseudosymmetric Weyl conformal curvature tensor. We mention that quasi-Einstein and 2-quasi-Einstein hypersurfaces with pseudosymmetric Weyl conformal curvature tensor immersed isometrically in semi-Riemannian spaces of constant curvature were investigated in the paper [33] (cited in [5]) and [4, respectively.

The above presented theorems together with Theorem 4.2 and Example 4.1 of the paper [68] (cited in [5]) imply

Theorem 0.3. Let $(\bar{M}, \bar{g})$ be a manifold which is isometric with a quasi-umbilical hypersurface immersed isometrically in a semi-Euclidean space $N$, $\operatorname{dim} N \geq 5$. Let $(\widetilde{N}, \widetilde{g})$ be a one-dimensional manifold. Then the manifold $(\bar{M} \times \tilde{N}, g=\bar{g} \times \widetilde{g})$ is a manifold with pseudosymmetric Weyl conformal curvature tensor satisfying $R \cdot R=$ $Q(S, R)$. Moreover, on the subset $U_{C}$ of this manifold we have $\operatorname{rank}(S-\rho g) \leq 2$, where $\rho$ is some function on $U_{C}$. 
Examples. (i) Let $\bar{S}$ and $\bar{\kappa}$ be the Ricci tensor and the scalar curvature of a Riemannian manifold $(\bar{M}, \bar{g}), \operatorname{dim} \bar{M}=3$. Moreover, let $\bar{S}$ has at every point of $\bar{M}$ exactly two distinct eigenvalues $\rho_{1}=\rho_{2}=\rho$ and $\rho_{3}=\bar{\kappa}-2 \rho$. Thus at every point of $\bar{M}$, we have $\operatorname{rank}(\bar{S}-\rho \bar{g})=1$. We mention that 3-dimensional Riemannian manifolds with the Ricci tensor having two distinct eigenvalues were investigated among others in 66, 7]. We consider now the product manifold $(M, g)=(\bar{M} \times \mathbb{R}, g=\bar{g} \times \widetilde{g})$, where $\widetilde{g}$ is the standard metric on $\mathbb{R}$. Let $S$ and $\kappa$ be the Ricci tensor and the scalar curvature of $(M, g)$, respectively. If we set $L_{C}=\frac{1}{2}\left(\frac{\kappa}{3}-\rho\right)$ then we get on $M$ : $\operatorname{rank}(S-\rho g)=\operatorname{rank}\left(S-\left(\frac{\kappa}{3}-2 L_{C}\right) g\right)=2$, provided that $\rho$ is nonzero at every point of $M$. Thus, in view of the presented above results, $(M, g)$ is a manifold with pseudosymmetric Weyl conformal curvature tensor and $C \cdot C=L_{C} Q(g, C)$ on $M$. (ii) Let $(\bar{M}, \bar{g}), \operatorname{dim} \bar{M} \geq 4$, be a Riemannian manifold defined in [1, Sec. 5(a.ii)]. That manifold is a conformally flat manifold satisfying $\operatorname{rank} \bar{S}=1$ on $\bar{M}$ ([1, p. 160]). Thus, the product manifold of $(\bar{M}, \bar{g})$ and a one-dimensional manifold is a manifold with pseudosymmetric Weyl conformal curvature tensor and its Ricci tensor $S$ satisfies $\operatorname{rank} S=1$. (iii) In Example 4.1(i) given in the paper [31] (cited in [5]) an example of a conformally flat manifold $(\bar{M}, \bar{g}), \operatorname{dim} \bar{M} \geq 4$, satisfying $\operatorname{rank} \bar{S}=1$ and $\bar{\kappa}=0$ is given. (iv) In [2, Example 7.6(i)] (cf. [4, Remark 3.1(ii)]) an example of a 2-quasiEinstein hypersuface $M$ in the standard unit $(n+1)$-sphere $S^{n+1}(1)$ is given. Moreover, if $n \geq 4$ then $M$ is a manifold with pseudosymmetric Weyl conformal curvature tensor. (v) In [3, Theorem 6.1] it was stated that every warped product manifold $\bar{M} \times_{F} \widetilde{N}$ with a 2-dimensional base manifold $(\bar{M}, \bar{g})$ and a 2-dimensional manifold $(\tilde{N}, \widetilde{g})$ or an $(n-2)$-dimensional Einstein semi-Riemannian manifold $(\tilde{N}, \widetilde{g})$, when $n \geq 5$, and a warping function $F$ is a 2-quasi-Einstein manifold. In particular, if $(\widetilde{N}, \widetilde{g})$ is a space of constant curvature then $\bar{M} \times{ }_{F} \widetilde{N}$ is a manifold with pseudosymmetric Weyl conformal curvature tensor [3, Theorem 7.1]. (vi) The Robinson-Trautman metric is a 4-dimensional Lorentzian warped product manifold with 2-dimensional fiber of non-constant curvature. In [8] it was showed that the Robinson-Trautman metric is 2-quasi-Einstein manifold and a manifold with pseudosymmetric Weyl conformal curvature tensor. (vii) Recently, in [11] the curvature properties of Vaidya metric, which is a 4-dimensional warped product manifold with 2-dimensional fiber of constant curvature were studied. Among other things it was showed that the Vaidya metric is a Ricci simple manifold with pseudosymmetric Weyl conformal curvature tensor.

\section{Acknowledgments}

The first- and third-named authors are supported by a grant of the Wrocław University of Environmental and Life Sciences, Poland [WIKSiG/441/212/S]. The firstnamed author is also supported by a Grant of the Technische Universität Berlin (Germany). The fourth- and fifth-named authors gratefully acknowledge the financial support of CSIR, New Delhi, India [File no: 09/025(0194)/2010-EMR-I, Project F. No. 25(0171)/09/EMR-II]. 


\section{References}

[1] J. Deprez, W. Roter and L. Verstraelen, Conditions on the projective curvature tensor of conformally flat Riemannian manifolds, Kyungpook Math. J. 29 (1989) 153-166.

[2] R. Deszcz, M. Głogowska, M. Hotloś and G. Zafindratafa, Hypersurfaces in space forms satisfying some curvature conditions, J. Geom. Phys. 99 (2016) 218-231.

[3] R. Deszcz, M. Głogowska, J. Jełowicki and G. Zafindratafa, Curvature properties of some class of warped product manifolds, Int. J. Geom. Methods Mod. Phys. 13 (2016), Article ID: 1550135, $36 \mathrm{pp}$.

[4] R. Deszcz, M. Głogowska, M. Petrović-Torgašev, M. Prvanović and L. Verstraelen, Curvature properties of some class of minimal hypersurfaces in Euclidean spaces, Filomat 29 (2015) 479-492.

[5] R. Deszcz, M. Hotloś, J. Jełowicki, H. Kundu and A. A. Shaikh, Curvature properties of Gödel metric, Int. J. Geom. Methods Mod. Phys. 11 (2014), Article ID: 1450025, 20 pp.

[6] O. Kowalski, A classification of Riemannian 3-manifolds with constant principal Ricci curvatures $\rho_{1}=\rho_{2} \neq \rho_{3}$, Nagoya Math. J. 132 (1993) 1-36.

[7] O. Kowalski and M. Sekizawa, Local isometry classes of Riemannian 3-manifolds with constant Ricci eigenvalues $\rho_{1}=\rho_{2} \neq \rho_{3}>0$, Archivum Math. (Brno) 32 (1996) $137-145$.

[8] A. A. Shaikh, M. Ali and Z. Ahsan, Curvature properties of Robinson-Trautman metric, J. Geom. 109 (2018), https://doi.org/10.1007/s00022-018-0443-1.

[9] A. A. Shaikh, R. Deszcz, M. Hotloś, J. Jełowicki and H. Kundu, On pseudosymmetric manifolds, Publ. Math. Debrecen 86 (2015) 433-456.

[10] A. A. Shaikh and H. Kundu, On generalized Roter type manifolds, Kragujevac J. Math. 43(3) (2019) 471-493.

[11] A. A. Shaikh, H. Kundu and J. Sen, Curvature properties of Vaidya metric, to appear in Indian J. Math. (2018), arXiv:1710.06333v1 [math.DG]. 